\title{
Heartbeat: Evidence, Experts and Trustworthy Guidelines
}

Cardiology has embraced the concept of evidence-based medicine with professional societies publishing clinical guidelines that have transformed cardiovascular care over the past 30 years. However, our current approach to guideline development in no longer viable-we need a process that responds more rapidly to new evidence, is free from bias, includes patients in the process, is based on rigorous review of the evidence by experts in research methodology, and is disseminated to clinicians and patients via intuitive and accessible multilayered digital formats. As discussed in an editorial in this issue of Heart (see page 3), the $B M J$ has partnered with the MAGIC project to produce the first BMJ RapidRecs, addressing transcatheter aortic valve implantation (TAVI) for severe aortic stenosis in patients at lower surgical risk. ${ }^{1-4}$ I hope many of you will take the time to look at this new approach and provide your feedback and thoughts (figure 1).

Appropriate treatment with oral anticoagulation reduces the risk of stroke in patients with atrial fibrillation (AF). However, AF often is not diagnosed until after a stoke has occurred because many patients are asymptomatic and the arrhythmia may be intermittent. Given the increasing prevalence of $\mathrm{AF}$ with the aging of our population, we urgently need better screening methods for this diagnosis. ${ }^{5}$ Chan and Choy (see page 24) report community screening for AF in over 13 thousand Hong Kong citizens using a one-time (30-second-long) smartphonebased single lead ECG. In these volunteers with a mean age of 65 years, the overall prevalence of AF by clinical history and ECG was $8.5 \%$ with $1.8 \%$ detected by the smartphone screening ECG, suggesting that many with a clinical history have paroxysmal AF. Previously undiagnosed AF was detected in 101 patients $(0.8 \%$ of the total screened), 2/3 of whom were asymptomatic. Risk score assessment indicated that most of the patients with newly diagnosed AF would benefit from anticoagulant therapy (table 1 ).

In the accompanying editorial, Svennberg and Engdahl (see page 6) point out that the prevalence of AF increases with age, so that screening starting at age 65

Correspondence to Professor Catherine M Otto, Division of Cardiology, University of Washington, Seattle, WA 98195, USA; cmotto@u.washington.edu years is likely to be more effective than including all ages, as in the study by Chan and Choy. In addition, "There are other risk factors for incident AF besides age. The CHA2DS2-VASc score has been reported to correlate with risk of incident $\mathrm{AF}$ in patients without AF, as well as height and weight. A more precise identification of individuals with increased risk for incident AF, possibly with the use of biomarkers, could enhance AF screening in future".

In 200 patients with AS and a normal ejection fraction, there was good correlation between Doppler echo mean gradient (MG) and computed tomographic quantitation of aortic valve calcification density (AVCd) in patients with a trileaflet aortic valve but only a weak association in those with a congenital bicuspid valve (see page 32 ) (figure 2). In patients with a trileaflet aortic valve, the only independent determinant of MG was AVCd, whereas in bicuspid valve patients both age and AVCd predicted AS severity. Of note, some younger patients with a bicuspid aortic valve had only minimal leaflet calcification (figure 3).
Cartlidge, Pawade and Dweck comment: (see page 8) "These observations have important implications both with respect to the utility of CT calcium scoring and the likely pathobiology underlying valve narrowing. First, it suggests that CT calcium scoring is likely to be of clinical utility in the vast majority of patients that we encounter in the clinic: subjects with trileaflet valves and patients $>51$ years with a bicuspid valve. Moreover, it indicates that calcium is crucial to the pathophysiology of valve narrowing in these patients and therefore an important potential therapeutic target. However, it also implies that CT calcium scoring may grossly underestimate the severity of AS in young patients under 50 years with a bicuspid valve".

Guideline recommended care for patients with heart failure and reduced ejection fraction (HFrEF) improves clinical outcomes yet is challenging to implement uniformly across a healthcare system. Emdin and colleagues (see page 55) examined variation in hospital performance for heart failure management in the National Heart Failure Audit

Figure 1 BMJ RapidRecs publication with Infographics, showing key information underlying the recommendation and link to MAGICapp (Making GRADE the Irresistible Choice authoring and publication platform). SAVR, surgical aortic valve replacement; TAVI, transcutaneous aortic valve implantation. 
Table 1 Baseline characteristics of participants with AF detected by SL-ECG or self-reported AF versus those with no AF

\begin{tabular}{|c|c|c|c|c|}
\hline & $\begin{array}{l}\text { Total } \\
(n=9807)\end{array}$ & $\begin{array}{l}\text { AF detected by SL-ECG or } \\
\text { self-reported by participants } \\
\text { ( } n=1111)\end{array}$ & $\begin{array}{l}\text { No AF } \\
\left(n=8696^{*}\right)\end{array}$ & $\mathrm{p}$ Value \\
\hline Age (years) & $65.5 \pm 13.3$ & $70.5 \pm 11$ & $64.8 \pm 13.4$ & $<0.001$ \\
\hline $\operatorname{Sex}(F), n(\%)$ & 7058 (72) & $716(64.4)$ & $6342(72.9)$ & $<0.001$ \\
\hline Weight (kg) & $59 \pm 10.4$ & $59.9 \pm 10.4$ & $58.8 \pm 10.4$ & 0.001 \\
\hline Height $(\mathrm{cm})$ & $157.3 \pm 8.6$ & $158 \pm 9$ & $157.2 \pm 8.6$ & 0.009 \\
\hline $\mathrm{BMI}\left(\mathrm{kg} / \mathrm{m}^{2}\right)$ & $23.8 \pm 3.6$ & $24 \pm 3.5$ & $23.8 \pm 3.6$ & 0.076 \\
\hline Waist circumference $(\mathrm{cm})$ & $83.5 \pm 9.8$ & $85 \pm 9.6$ & $83.3 \pm 9.8$ & $<0.001$ \\
\hline \multicolumn{5}{|l|}{ Comorbid conditions } \\
\hline Hypertension, n (\%) & 3720 (37.9) & $421(37.9)$ & 3299 (37.9) & 1.0 \\
\hline Diabetes, n (\%) & $1515(15.4)$ & $232(20.9)$ & $1283(14.8)$ & $<0.001$ \\
\hline Hyperlipidaemia, n (\%) & $1934(19.7)$ & $332(29.9)$ & 1602 (18.4) & $<0.001$ \\
\hline Heart failure, $\mathrm{n}(\%)$ & $85(0.9)$ & $56(5)$ & $29(0.3)$ & $<0.001$ \\
\hline Stroke, n (\%) & $284(2.9)$ & $70(6.3)$ & $214(2.5)$ & $<0.001$ \\
\hline $\begin{array}{l}\text { Coronary artery disease, } \\
\mathrm{n}(\%)\end{array}$ & $206(2.1)$ & $89(8)$ & $117(1.3)$ & $<0.001$ \\
\hline $\begin{array}{l}\text { Valvular heart disease, } \\
\text { n (\%) }\end{array}$ & $84(0.9)$ & $47(4.2)$ & $37(0.4)$ & $<0.001$ \\
\hline $\begin{array}{l}\text { Peripheral vascular } \\
\text { disease, } \mathrm{n}(\%)\end{array}$ & $48(0.5)$ & $22(2)$ & $26(0.3)$ & $<0.001$ \\
\hline $\begin{array}{l}\text { Obstructive sleep apnoea, } \\
\text { n (\%) }\end{array}$ & $88(0.9)$ & $17(1.5)$ & $71(0.8)$ & 0.026 \\
\hline Thyroid disease, $\mathrm{n}(\%)$ & 366 (3.7) & $40(3.6)$ & $326(3.7)$ & 0.867 \\
\hline COPD, n (\%) & $37(0.4)$ & $10(0.9)$ & $27(0.3)$ & 0.007 \\
\hline $\begin{array}{l}\text { Cardiothoracic surgery, } \\
\mathrm{n}(\%)\end{array}$ & $266(2.7)$ & $107(9.6)$ & 159 (1.8) & $<0.001$ \\
\hline
\end{tabular}

*Participants who reported 'don't know' for a history of AF together with AF not detected by SL-ECG were excluded. $A F$, atrial fibrillation; BMI, body mass index; COPD, chronic obstructive pulmonary disease; $F$, female; SL-ECG, wireless single-lead ECG.

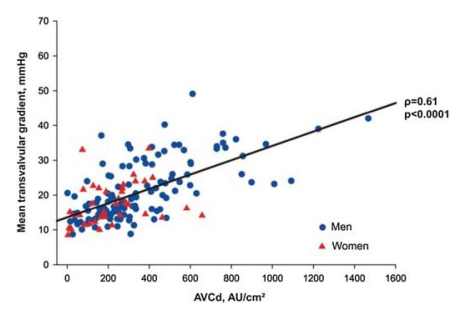

Figure 2 Correlation between the mean transvalvular gradient and the aortic valve calcification density (AVCd) in the tricuspid aortic valve subgroup $(n=161)$. Men are represented by blue dots and women are represented by red triangles.

for England \& Wales. Hospital adherence to key performance measures ranged from $50 \%$ to $97 \%$ with only $8 \%$ (95\% CI $7 \%$ to $10 \%)$ of this variation attributable to variations in hospital features. Adjusted hospital-attributable variation in prescription rates for recommended heart failure medications was low (average 7\%) with larger variations seen in referral to specialist follow-up (median $81 \%$, range $20 \%$ to $100 \%$ ) with $26 \%$ of this being attributable to hospital-level differences (CI 22\% to 31\%). The authors conclude: "While further investment into costly organisational changes for management of HFrEF

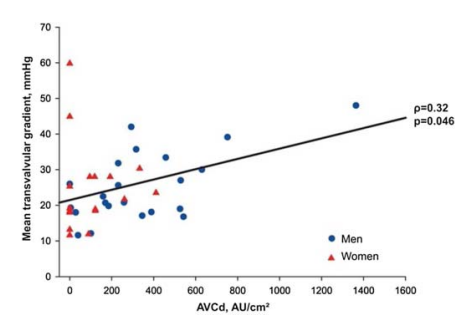

Figure 3 Correlation between the mean transvalvular gradient and the aortic valve calcification density (AVCd) in the bicuspid aortic valve subgroup $(n=39)$. Men are represented by blue dots and women are represented by red triangles.

in hospitals in England and Wales may still be useful for changing other important healthcare outcomes across hospitals, our study shows that such investments cannot be expected to lead to large reductions in variability in hospital adherence to heart failure performance measures examined in this study. Future healthcare delivery efforts should consider evaluation and improvement of more ambitious key performance measures".

Our series on Graphics and Statistics for Cardiology continues in this issue with a detailed discussion of graphical

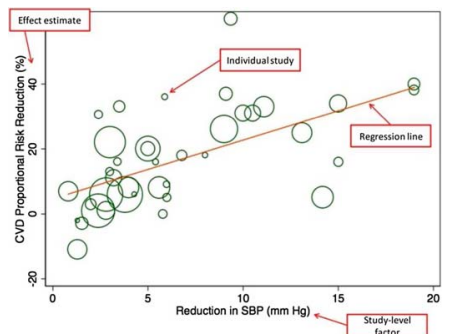

Figure 4 Example of meta-regression plot: meta-regression plot of the percentage risk reduction in major cardiovascular disease (CVD) events regressed against the difference in achieved systolic blood pressure (SBP) between treatment arms.

display of data in a meta-analysis paper (see page 19). The key graphics that are discussed and illustrated are a flow diagram summarizing identification of included studies, the optimal format for a forest plot, displays for the evaluation of study biases, meta-regression bubble plots and a checklist for producing optimal graphics (figure 4). We encourage authors to follow these recommendations for any meta-analysis papers submitted to Heart.

The Education in Heart article in this issue by Prof. Olaf Wendler (see page 78) summarizes the approach to diagnosis, medical and surgical therapy for aortic dissection. The Image Challenge (see page 18) shows a case of a cardiac mass in an infant, with a discussion of the differential diagnosis for this imaging finding.

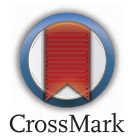

To cite Otto CM. Heart 2017;103:1-2.

Heart 2017:103:1-2.

doi:10.1136/heartjnl-2016-310947

\section{REFERENCES}

1 Vandvik PO, Otto CM, Siemieniuk RA, et al. Transcatheter or surgical aortic valve replacement for patients with severe, symptomatic, aortic stenosis at low to intermediate surgical risk: a clinical practice guideline. BMJ 2016;354:i5085.

2 Siemieniuk RA, Agoritsas T, Manja V, et al. Transcatheter versus surgical aortic valve replacement in patients with severe aortic stenosis at low and intermediate risk: systematic review and meta-analysis. BMJ 2016;354:15130.

3 Foroutan F, Guyatt GH, O'Brien K, et al. Prognosis after surgical replacement with a bioprosthetic aortic valve in patients with severe symptomatic aortic stenosis: systematic review of observational studies. BMJ 2016;354:15065.

4 MagicAPP webiste. Improving patient care through guidelines, evidence summaries and decision aids we can all trust, use and share. https://www.magicapp. org/ Accessed November 15, 2016.

5 Keach JW, Bradley SM, Turakhia MP, et al.Early detection of occult atrial fibrillation and stroke prevention. Heart 2015;101:1097-102 